\title{
Corrigendum: Regulation of cardiovascular development and integrity by the heart of glass-cerebral cavernous malformation protein pathway
}

Benjamin Kleaveland, Xiangjian Zheng, Jian J Liu, Yannick Blum, Jennifer J Tung, Zhiying Zou, Shawn M Sweeney, Mei Chen, Lili Guo, Minmin Lu, Diane Zhou, Jan Kitajewski, Markus Affolter, Mark H Ginsberg \& Mark L Kahn

Nat. Med. 15, 169-176 (2009); published online 18 January 2009; corrected after print 12 February 2009

In the version of this article initially published, Shawn M. Sweeney was not included in the list of authors. The error has been corrected in the HTML and PDF versions of the article.

\section{Corrigendum: A pivotal role for galectin-1 in fetomaternal tolerance}

Sandra M Blois, Juan M Ilarregui, Mareike Tometten, Mariana Garcia, Arif S Orsal, Rosalia Cordo-Russo, Marta A Toscano, Germán A Bianco, Peter Kobelt, Bori Handjiski, Irene Tirado, Udo R Markert, Burghard F Klapp, Francoise Poirier, Julia Szekeres-Bartho, Gabriel A Rabinovich \& Petra C Arck

Nat. Med. 13, 1450-1457 (2007); published online 18 November 2007; corrected after print 7 May 2009

In the version of this article initially published, the plot labeled "Stress + Gal-1" duplicated the plot labeled "Control” for the IL-12p70 staining in Figure 2f. The corrected plots have now been provided in the HTML and PDF versions of the article. Two sentences were omitted from the section on purification of uterine DCs in the Methods. The sentences should have read: "We obtained purified DCs from uterine tissue in very low numbers. Thus, we pooled isolated cells from each group and used this cell cocktail for the isotype control staining." The error has been corrected in the HTML and PDF versions of the article. In Figure 4f, the lanes of the western blot were merged inappropriately. The properly presented blot, in which the gel lanes have been separated to indicate that the samples were not originally run side by side, has been provided in the PDF and HTML versions of the article. The standard curve of the cytometric bead array kit used to generate the cytokine data can be found in the revised Supplementary information available online. 